\title{
Data encoding and reconstruction of thermal imaging maps of impact damaged composite Structures using feature space and neural networks
}

\author{
Mahmoud Iskandarani \\ Faculty of Engineering, Al-Ahliyya Amman University, 19328, Amman, Jordan
}

\begin{abstract}
A new approach to characterizing and predicting impact damage level in (Reaction Injection Molding) RIM structures is presented. The technique encodes thermal images maps and extracts features from presented thermal images. Complex Neural Networks structure is employed to reconstruct thermal imaging maps and predict the extent of damage an impact can cause. Neural network weigh elimination algorithm is used and proved effective in predicting areas of damage.
\end{abstract}

\section{Introduction}

Structural Integrity Monitoring (SIM) is the ability to detect and classify structural changes that have an adverse effect on the performance of the component. The purpose is to improve reliability and life-cycle. In some industries, damage can lead to catastrophic consequences, which is critical in many commercial and military applications that employ composite materials to take advantage of their excellent specific strength and stiffness properties, and fatigue performance.

Composite materials are hard to design, manufacture, and repair compared to other materials since they tend to fail through diverse interacting damage modes. In addition, damage detection in composites is more difficult in other structures due to the structural characteristics of the composed Matrix-Fiber. Traditional non-destructive testing (NDT) techniques are Impractical for efficient design and in-service testing. Thus, it is essential to not only develop new testing techniques but also advanced interpretation algorithms.

Thermal non-destructive testing is used to detect damage in composites due difference in heat transfer properties between damaged and undamaged regions within the tested structure. When external heat is applied, the presence of defects affect the normal heat flow pattern of the structure. If this heat propagation is altered sufficiently, a temperature distribution profile can be realized. This distribution is then related to the damage.

Infrared thermography is widely used in many NDT applications to detect for example crack, corrosion and surface scratches. It is a rapid testing technique as it has a short testing time, which covers large testing area with observable testing results. Infrared image show defects, which through specific algorithms can be classified. In addition, the ability to obtain higher definition and contrast images will facilitate better analysis and computation [1-14]

Artificial Neural network can map the critical properties of materials without the need for a computational mathematical model. Different researchers designed and applied neural network models and algorithms to characterize, and predict materials variables such as, strain and stress. Such effects results from impact damage and would change certain features in the testing image of a damaged sample compared to the one before impact. The new captured image will display a pattern which differ from a reference image texture, color or intensity, among others and can be used to establish level and magnitude of damage and component validity as a function of feature distribution change [15-23].

In this paper, a new method is presented that incorporates an optimized by neural networks algorithm for detecting and recognizing damage in RIM structures changes tested with thermal energy flow. The method and technique is used to construct feature maps as a function of impact energy.

\section{Materials and Methods}

Each chosen composite was a laminate made by Resin Injection Molding (RIM) to produce $5 \mathrm{~mm}$ thick samples. Pulse video thermography (PVT) is employed with Xenon heat source and thermal image capturing system. Images are taken for all tested components. The resulting images mapped and encoded into arrays of data to represent extracted features. The reference encoding feature map is shown in Figure 1.

*orresponding author: $\underline{\text { m.iskandarani@ammanu.edu.jo }}$ 


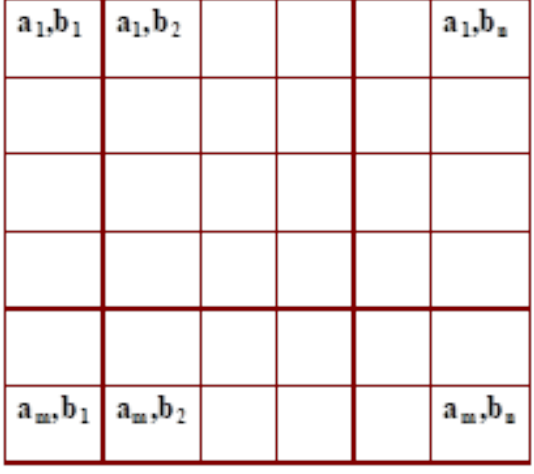

Fig. 1. Encoding Map.

Weight Elimination Algorithm (WE) is used to carry out neural network training and prediction. Its function based on error minimization by using a cost term that is added to the original error function.

The cost term in weight decay affects large weights, as it causes weights under consideration to converge to smaller values. If the weights with large values are between input layer and hidden layer, they can result in the output function with possible discontinuities.

However, if large weights lie between the hidden layer and the output layer, they can result in outputs outside the range of the data. Hence, large weights can cause significant variance of the output and destabilize the neural structure.

The overall weight elimination error function is presented by equation (1), and it consists of two parts represented in the overall expression in equation (1):

I. Initial error function described by equation (2).

II. Cost error function described by equation (3)

$$
\begin{gathered}
E_{w}=E_{\text {Initial }}+E_{\text {cost } \ldots(1)} \\
E_{\text {Initial }}=\frac{1}{2} \sum_{k}\left(d_{k}-o_{k}\right)^{2} \ldots(2) \\
E_{\cos t}=\mu\left(\sum_{j k} \frac{\left(\frac{w_{j k}}{w_{\text {neural }}}\right)^{2}}{1+\left(\frac{w_{j k}}{w_{\text {neural }}}\right)^{2}}\right) \ldots(
\end{gathered}
$$

Where;

$E_{w}$ : The combined overhead function that includes the initial overhead function, $E_{\text {Initial }}$ and the weightelimination term $E_{\cos t}$. $\mu$ : The weight-reduction factor,

$w_{j k}$ : Represents the individual weights of the neural network model

$w_{\text {neural }}$ : A scale parameter computed by the WEA.

$d_{k}:$ The desired Output

$o_{k}:$ The actual Output

The dynamic weight changes is calculated through a modified version of the gradient descent algorithm as shown in equation (4)

$$
\Delta w_{j k}=\left(-\eta \frac{\partial E_{\text {Initial }}}{\partial w_{j k}}\right)-\left(\mu \frac{\partial E_{\cos t}}{\partial w_{j k}}\right) \ldots
$$

Where;

\section{$\eta$ : The Learning Rate (between 0 and 1)}

The parameter, , is a scale parameter computed by the WEA, and selected to be the smallest weight from the last set of epochs to force small weights to zero.

$w_{\text {neural }}$ Guides the computing algorithm to find solutions with either smaller number of large weights or larger number of small weights.

\section{Analysis and Discussion}

Figures 2 to 4 show thermal feature maps for captured images of RIM composite structures.

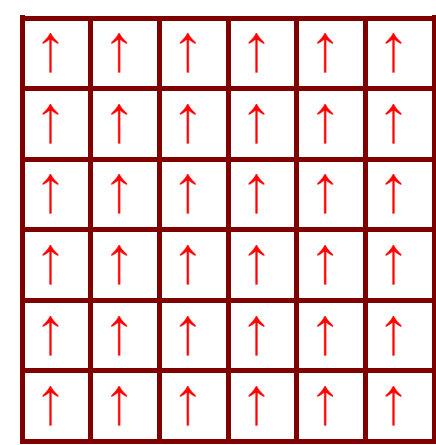

Fig. 2: Thermal image map of reference composite sample (0J) 


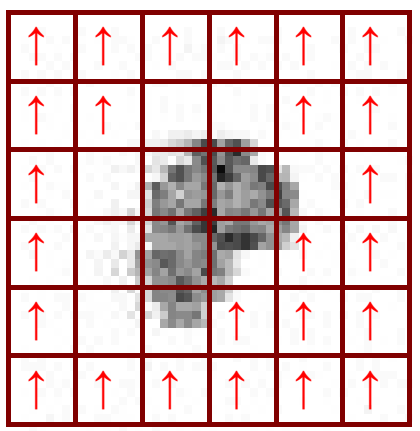

Fig. 3: Thermal Image map of $30 \mathrm{~J}$ impact damaged composite sample

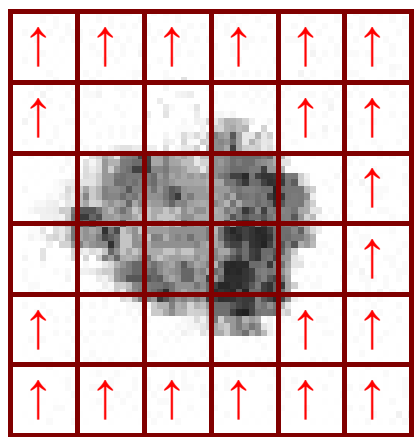

Fig. 4: Thermal Image map of $40 \mathrm{~J}$ impact damaged composite sample

Figure 5 shows the neural network simulation system using Weight Elimination Algorithm, while Figure 6 shows the Neural Model used to map the obtained thermal images into features and to predict the extent of damage for similar samples if subjected to other magnitudes of impact.

Figures 7 to 9 show three of the predicted encoding maps, where ones indicates no damage and zeros indicates the presence of damage with Table 1 showing training and predicted values.

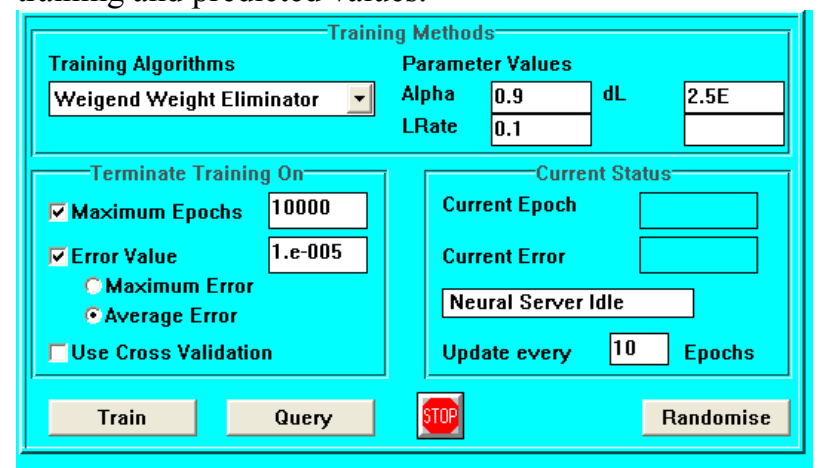

Fig. 6: Neural networks simulation system

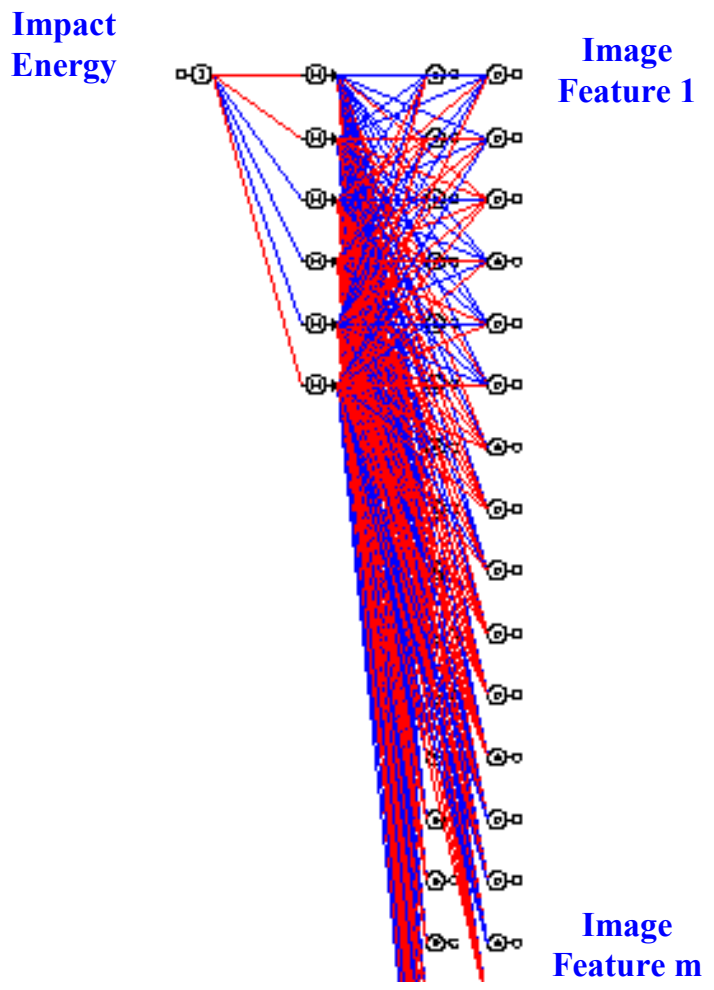

Fig. 6: Neural Network complex structure used for mapping and predicting damage in tested RIM composites.

\begin{tabular}{|l|l|l|l|l|l|}
\hline 1 & 1 & 1 & 1 & 1 & 1 \\
\hline 1 & 1 & 1 & 1 & 1 & 1 \\
\hline 1 & 1 & 1 & 1 & 1 & 1 \\
\hline 1 & 1 & 1 & 1 & 1 & 1 \\
\hline 1 & 1 & 1 & 1 & 1 & 1 \\
\hline 1 & 1 & 1 & 1 & 1 & 1 \\
\hline
\end{tabular}

Fig. 7: 0J predicted thermal encoding map

\begin{tabular}{|l|l|l|l|l|l|}
\hline 1 & 1 & 1 & 1 & 1 & 1 \\
\hline 1 & 1 & 0 & 0 & 1 & 1 \\
\hline 1 & 0 & 0 & 0 & 0 & 1 \\
\hline 1 & 0 & 0 & 0 & 1 & 1 \\
\hline 1 & 0 & 0 & 1 & 1 & 1 \\
\hline 1 & 1 & 1 & 1 & 1 & 1 \\
\hline
\end{tabular}

Fig. 8: 30J predicted thermal encoding map 


\begin{tabular}{|l|l|l|l|l|l|}
\hline 1 & 1 & 1 & 1 & 1 & 1 \\
\hline 1 & 0 & 0 & 0 & 0 & 0 \\
\hline 0 & 0 & 0 & 0 & 0 & 0 \\
\hline 0 & 0 & 0 & 0 & 0 & 0 \\
\hline 1 & 0 & 0 & 0 & 0 & 0 \\
\hline 1 & 1 & 1 & 1 & 1 & 1 \\
\hline
\end{tabular}

Fig. 9: 60J predicted thermal image map

Figure 10 shows a representation plot that correlates impact energy to feature loss. From the plot, it is clear that there are three main stages or phases of damage:

1. Low impact Energies $(0 \mathrm{~J}<$ Impact Energy $<20 \mathrm{~J})$ :

The composite structure suffered a minor surface scratches with no real propagation of damage through the Matrix-Fiber System.

\section{Medium Impact Damage $\quad(20 \mathrm{~J}<$ Impact Energy $<40 \mathrm{~J})$}

The composite structure suffered external and internal damage with the Matrix-Fiber system still intact.

\section{High Impact Damage (Impact Energy $>40 J)$ :}

The composite component suffered deep internal damage that resulted in damaging the Matrix-Fiber system, with fiber breakage and fiber pullout, hence, the component can no longer be used in service.

The impact causes both lateral and longitude waves to propagate through the composite structure, thus the damage surface area should also increase in relation to increased impact energy. Subjecting a RIM composite structure to successive impacts will have an accumulative effect and the area of low impact would expect to shift towards the medium one and the medium one towards the high one if the component is subjected to successive incidents of damage.

Table 1. Predicted Space Features

\begin{tabular}{|c|c|c|}
\hline $\begin{array}{c}\text { Impact } \\
\text { Energy } \\
\mathbf{J}\end{array}$ & $\begin{array}{c}\text { Training Affected } \\
\text { Space Features }\end{array}$ & $\begin{array}{c}\text { Predicted Affected } \\
\text { Space Features }\end{array}$ \\
\hline 0 & 0 & 0 \\
\hline 10 & 4 & 5 \\
\hline 20 & 8 & 9 \\
\hline 30 & 10 & 11 \\
\hline 40 & 13 & 14 \\
\hline 50 & 20 & 19 \\
\hline 60 & 23 & 22 \\
\hline
\end{tabular}

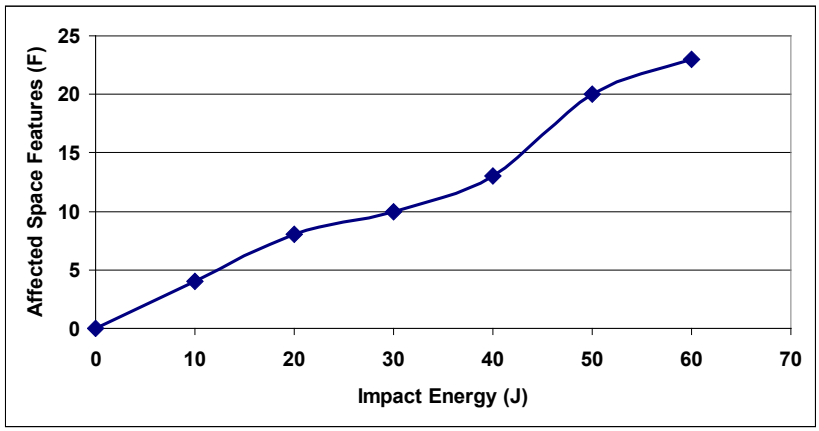

Fig. 10: Relationship between Impact Energy and Affected Space Features

The analyzed and reconstructed images contain two sections as represented in equation (5):

$$
\operatorname{Im} \text { age }=\left(\left(\sum_{L=0}^{255} \text { Healthy Section }(\text { Affected Features })\right),\left(\sum_{L=0}^{255} \text { Damaged Section }(\text { Affected Features })\right)\right) \ldots(5)
$$

Equation (5) can be further represented in relation to Affected Features as shown in equation (6);

$$
\text { Im age }=\left(\left[\sum_{\text {Affected Features }=0}^{N}\left(L_{0}+L_{128}+L_{180}\right)\right],\left[L_{255}\right]\right) \ldots
$$

Where;

$\mathrm{N}$ : Maximum number of Affected Features as a function of Impact Energy.

Applying equation (6) to the tested composites at impact energies of $30 \mathrm{~J}, 40 \mathrm{~J}$, results in equations (7), (8):

$$
\begin{aligned}
& \operatorname{Im} \text { age }(30 J)=\left(\left[\sum_{\text {Affected Features }=0}^{10}\left(L_{0}+L_{128}+L_{180}\right)\right],\left[L_{255}\right]\right) \ldots \\
& \operatorname{Im} \text { age }(40 J)=\left(\left[\sum_{\text {Affected Features }=0}^{13}\left(L_{0}+L_{128}+L_{180}\right)\right],\left[L_{255}\right]\right) \ldots
\end{aligned}
$$

\section{Conclusions}

The reconstruction of maps for the RIM composite damaged surface using Neural Networks is an excellent step towards a smart image simulation system that can be used during the design and manufacturing of composite structures. It can be integrated within the prototyping process. Also, it can be used for testing of damaged structures to enable reporting on the ability of the affected component to stand more impacts if not replaced or repaired, which is a function of the area of application and how critical and frequent impacts are.

\section{References}

1. M. Iskandarani, Journal of Computer Science 14, 6 (2018) 
2. M. Iskandarani, International Journal of Advanced Computer Science and Applications 9, 6 (2018)

3. M. Iskandarani, Journal of Computer Science 13, 9 (2017)

4. M. Iskandarani, Engineering 8, 4 (2016)

5. R.Usmantiagar, P. Venegas, J. Guerediaga, L. Vega, and I. Lopez, NDT \& E International 54 (2013)

6. A. Katunin, P. Przystałka, J. Guerediaga, and A. Wronkowicz, Machine Dynamics Research 38, 3 (2014)

7. L. Ma, D. Liu, International Journal of Imaging Systems and Technology 50, 22 (2016)

8. F.Wang, J. Liu, Y. Liu, Y. Wan, International Journal of Imaging Systems and Technology 84 (2016)

9. T.Liang, W. Ren, G. Tian, M. Elradi, Y. Gao, Composite Structure 143 (2016)

10. R.Yang, Y. He, H. Zhang, Renewable and Sustainable Energy Reviews $\mathbf{6 0}$ (2016)

11. D. Palumbo, R. De Finis, G.Demelio, U. Galietti, Composite Part B, 103 (2016)

12. D. Palumbo, R. De Finis, G. Demelio, U. Galietti, Composite Part B, 117 (2017)

13. D. Balageas, J. Roche. H. Leroy, Materials Evaluation 75, 8 (2017)

14. M. Iskandarani, American Journal of Applied Sciences 10, 2 (2013)

15. M. Iskandarani, Journal of Intelligent Learning Systems and Applications n5 (2015)

16. H. Balcıŏglu, A. Seçkin, and M. Aktaş, Journal of Composite Materials n50, 23 (2016)

17. T.Hessamodin, A. Milani, R. Seethaler, and A. Heidarzadeh, Open Journal of Composite Materials 6 (2016)

18. P. Rajendran, S. Srinivasan, Strain 52, 1 (2015)

19. F.Peng, J. Li, M. Long, Journal of Forensic Sciences 60, 2 (2015)

20. B.Kumar, R. Harikumar, Journal of Imaging Systems and Technology 25, 1 (2015)

21. H.Dai, H. Zhang, and W. Wang, Computer-Aided Civil and Infrastructure Engineering 30, 2 (2015)

22. T.Barry, M. Kisharaju, C. Nagarajah, S. palanisamy, ournal of Composite Materials 50, 7 (2016)

23. B.Ashwini, B. Yuvaraju, Research in Computer and Communication Engineering 4, 4 (2016) 